\title{
Bases para un sistema de información para el estudio de la historia de la lectura en Extremadura
}

\author{
Lying the ground for an Information System on the History of Reading in Extremadura
}

\author{
Aitana Martos García (1) y Agustín Vivas Moreno (2) \\ (1) Universidad de Extremadura. Facultad de Biblioteconomía y Documentación. amar- \\ gar@alcazaba.unex.es. (2) avivas@alcazaba.unex.es.
}

\begin{abstract}
Resumen
La siguiente comunicación tiene por objeto el estudio de la historia de la lectura a través de la aplicación de las técnicas documentales, tales como la recuperación de las fuentes de información (directas e indirectas), la confección de métodos de análisis de contenido conceptual y la extracción, a través de ellas, de instrumentos de recuperación de información tales como tesauros y diversas herramientas documentales conceptuales. La confluencia de éstas en un sistema documental automatizado como el que proponemos, nos proporcionaría innegables beneficios para poder rastrear manifestaciones de la historia del libro y de la lectura en las múltiples y variadas fuentes de información, arrojando pertinencia y efectividad en la recuperación demandada. En consecuencia, mediante este estudio se pretende sentar las bases para la creación de un sistema de información documentalpara el estudio de la historia del libro y de la lectura en Extremadura.
\end{abstract}

Palabras clave: Cultura escrita. Extremadura (España). Herramientas documentales. Historia del Libro. Historia de la Lectura. Sistemas de Información.

\section{Introducción}

El presente trabajo de investigación intenta ser una primera aproximación al establecimiento de un sistema de información documental para el estudio de la historia del libro y la lectura en Extremadura. Este estudio supondrá el conocimiento de diversos aspectos históricos y socioculturales sobre el tema, los cuales se tomarán en cuenta como base en subsiguientes estudios sobre esta área.

En consecuencia, la meta general de este trabajo, a grandes rasgos, será sentar las bases teóricas y técnicas para la confección de dicho sistema de información. Nuestro objetivo principal, por tanto, será proporcionar al usuario una serie de procedimientos y pautas para emprender la creación de un Sistema de Información

\begin{abstract}
This work intends to study the History of Reading through documentary techniques, as information retrieval from direct and indirect sources, making up methods of conceptual content analysis, and creation of tools for information retrieval as thesaurus and others. The junction of all of them in a automated documentary management system, would provide undeniable benefits to find History of Books and Reading signs in many information sources, giving pertinence and effectiveness in the retrieval. Therefore, with this study we intend to lay the foundations for the creation of a documentary information system for the study of History of Books and Reading in Extremadura.
\end{abstract}

Keywords: Written Culture. Extremadura. Management tools. History of the Book. History of Reading. Information systems.

que nosotros hemos concebido para el estudio de la historia del libro y la lectura en Extremadura en concreto ( $y$ que luego ampliaríamos a un espectro más amplio del campo que sería la Cultura Escrita en la región), pero que en general se puede extrapolar para el estudio de cualquier materia cultural-histórica. No entraremos en detalles sobre los pormenores técnicos o de utilización de una aplicación que por ahora es meramente teórica, pero que puede utilizarse para llevar a cabo futuras investigaciones.

De todo ello, se desprenden los cometidos principales de esta investigación:

i. Crear una herramienta que sirva de base para el estudio de la historia del libro y la lectura en Extremadura como disciplina, a través de la 
rigurosa revisión de la historiografía existente, y determinar sus perspectivas de futuro.

ii. Justificar la utilidad de la aplicación de las técnicas documentales al análisis de la historia del libro y la lectura. Esto es, aplicar distintas técnicas documentales de análisis de contenido a diversa documentación, desarrollando ciertas herramientas documentales que permitan entresacar el contenido relativo la materia.

iii. Sentar bases documentales para la creación de un sistema de información dotado del mayor rigor y exhaustividad científica posibles para la investigación de aspectos relativos a la historia del libro y la lectura en Extremadura.

En definitiva, este estudio estaría confeccionado mediante el análisis riguroso y profundo de la documentación vinculada a la historia del libro y la lectura como base, pero tiene como propósito sentar unos fundamentos para un sistema de información mediante el que sea posible localizar, catalogar y analizar dicha documentación. En el desarrollo metodológico del trabajo se emplearán técnicas documentales (que darán como resultado la creación de un catálogo documental y un tesauro ad hoc como lenguaje documental específico para la temática), el análisis crítico de la diversa documentación empleada, y el uso de las nuevas tecnologías de la información (que darán lugar a la confección un gestor documental automatizado aplicable a la temática en cuestión, y que permitirá la consulta y recuperación de la formación de forma pertinente y eficaz).

\section{Contextualización del objeto de investigación}

\subsection{La Historia de la Lectura y el Libro}

Desde hace aproximadamente medio siglo, la historia del libro se ha afianzado en la investigación internacional como una disciplina que abarca -al decir de los teóricos franceses esencialmente- varios enfoques diferentes y complementarios (1):

1. Por un lado, el libro es una mercancía que se produce y se vende, y, en consecuencia, su estudio puede estar sometido a dos líneas de investigación: por un lado, a los métodos de la profusa historia economicista, interesada en el estudio de la coyuntura de la producción impresa, la geografía de los talleres o las áreas comerciales; y por otro, a los múltiples procedimientos de la historia social concerniente a los encargados de la producción y venta de libros (aprendices, obreros canjistas, etc.), sus contratos y obligaciones, o los hábitos de trabajo. Se trata, por tanto de la aplicación de la historia cuantitativa, repleta de fuentes tales como libros de cuentas, libros de pagos, encuestas administrativas, registros de permisos de impresión, etc.

2. En segundo lugar, la historia del libro puede estudiarse desde lo que el Diccionario del saber moderno de los autores franceses viene denominando como su bibliografía material, esto es, aquella perspectiva sustancialmente técnica que tiene que ver con el conocimiento del proceso de fabricación del libro antiguo, la realización de bibliografías científicas -distinguiendo, por ejemplo, ediciones diferentes de un mismo texto y diferentes estados de una misma edición- el descubrimiento de falsificaciones e incluso, la historia del funcionamiento de los distintos talleres y sus utensilios, describiendo técnicamente las operaciones que se llevaban a cabo en el minucioso proceso de impresión.

3. Por último, existe un tercer enfoque que quizás sea el más interesante. Aquél que analiza al libro como conductor de texto y que tiene por objeto analizar la producción y la difusión de la cultura del libro. Esta perspectiva presenta diferentes panorámicas:

3.1. En primer lugar, el libro es una provechoso instrumento para determinar todo cuanto una sociedad ha pensado o escrito. Así, por ejemplo, se han realizado abundantes historias seriadas para reconstituir el conjunto de libros producidos en un tiempo y en un lugar determinados (series de libros impresos, por materias, por autores, etc.); y, al mismo tiempo, se han estudiado diferentes espacios regionales, que permiten individualizar las desiguales conductas en la producción del libro.

3.2. Asimismo, a través del libro estudiamos a sus autores y sus múltiples vinculaciones. La condición de autor, en sus relaciones con los libreros y editores, con los poderes -y por tanto con las diferentes censuras-, con las instituciones de cultura es, en efecto, uno de los datos fundamentales para comprender las condiciones de la producción de lo impreso.

3.3. Por último, el libro es un magnífico prontuario para una sociología de la lectura. Se trata de uno de los temas de más actualidad, entrecruzándose en su estudio múltiples cuestiones diferentes, tales como el problema del acceso al libro - que no pasa forzosamente por la compra, dado que puede pasar por mediación de lo oral (lectura en voz alta para otro) o por el uso de una colección abierta al público- o el estudio de las lecturas supuestas en cada clase social, para extraer así el perfil cultural o ideológico de cada uno de ellas 
Ahora bien ¿Qué hay realizado en Extremadura sobre la historia de la lectura? Ciertamente si para la historia del libro hay poco hecho en Extremadura, para la historia de la lectura el desierto historiográfico es casi total, con apenas algún título especifico sobre la temática en cuestión. Veamos algunos puntos:

1. La obra más representativa para el ámbito extremeño sea quizás la de Ricardo Luengo Pacheco para Plasencia, analizando casi 10.000 libros de 134 bibliotecas, de tres siglos de historia cultural placentina (siglos XVI-XVIII), examinando para ello más de 400 protocolos notariales. Se trata, que sepamos, del único intento delimitado al tema, preguntándose por los poseedores, sus profesiones, la forma de adquisición de los libros o los espacios de la lectura (locales, mobiliarios y decoración) (Luengo Pacheco, 2002).

2. Ciertamente contamos con algunas obras que tocan tangencialmente el estudio del libro y de sus lectores, fundamentalmente provenientes de estudios demográfico-sociales y de las mentalidades. Se trata de estudios que tienen por objeto cuestiones diversas que tienen en algún momento como telón de fondo el examen de cuestiones vinculadas. Es el caso, entre otros, de Testón Núñez y su análisis de las bibliotecas de algunos personajes cacereños para el análisis de la mentalidad del s. XVII, de Santillana Pérez y su examen de la vida y muerte en el partido de Cáceres en el s. XVIII, de Aragón Mateos que analiza los gustos literarios de la nobleza extremeña en el s. XVIII, de Fernando Marcos Álvarez y Fernando Cortés que centraron sus indagaciones en la política educativa y el analfabetismo del s. XVII y la alfabetización militar y las lecturas de los soldados del Real Ejército de Extremadura entre 1640 y 1668, dada la Guerra de Secesión-Restauraçao portuguesa, o de Vivas Moreno poniendo énfasis en la necesidad de la historia de la lectura para la Extremadura del Antiguo Régimen (2).

3. Por otro lado, otros asuntos han sido tratado de forma discontinua. Así, los niveles de alfabetización durante la Edad Moderna, un tema vinculado de alguna forma a la evolución de la lectura, es acotado parcialmente por Le Flem para Plasencia, Cáceres y Trujillo mediante el análisis de fuentes fiscales, el caso de la censura de libros por la inquisición, estudiado por Fernández Nieva, o la educación y la instrucción pública en Extremadura, por parte de Santillana Pérez o Rodríguez Cancho (3).

En definitiva, el desierto historiográfico resulta prácticamente enterizo. Bien es cierto que Arias Montano, Bartolomé José Gallardo, el Marqués de Jerez, Rodríguez Moñino o Manuel Rozas han dado categoría, desde Extremadura, a la bibliofilia hispana. Sin embargo, la historia del libro y de la lectura en Extremadura se caracteriza por la casi total ausencia de obras monográficas al respecto. Contamos, en este sentido, con multitud de fuentes directas sin explotar base de investigaciones científicas. Las obras de que disponemos son heterogéneas, con métodos y fuentes desiguales, y con objetos de estudios diversos, con especial atención a los de carácter educativo.

Llegados a este punto, podemos preguntarnos qué pretendemos cuando iniciamos un proyecto de sistema de información sobre la historia de la lectura en Extremadura. Al respecto, M. Chevalier (1976) hablaba de tres fenómenos distintos para los cuales nuestro sistema de información ofrecería cuantiosas funciones:

1. Uno de carácter sociocultural: quién sabe leer, siendo conscientes de la dificultad que entraña la distinción entre alfabetos y analfabetos, hasta tal punto que en la mayoría de las veces la determinación viene dada por si sabe o no firmar.

2. Otro de carácter socioeconómico: quién tiene la posibilidad de leer. El precio de los libros, pero también las almonedas, la disposición de acceso a bibliotecas privadas por parte de criados y mayordomos o la costumbre de prestarse libros y manuscritos tendrían cabida aquí.

3. Y un último, de carácter puramente cultural: quién llega a adquirir la práctica de la lectura. En este orden de cosas, el interés viene marcado por la posibilidad. Bien es verdad, que todo parece indicar que en la Extremadura del Antiguo Régimen, el público que realmente tenía interés por la lectura, es un público muy reducido - tal y como sucedía en el resto de España salvo honrosas excepciones- como lo demuestra la ausencia de grandes bibliotecas privadas, el menguado interés de los pocos mercaderes de la época por la cultura, o la escasa inclinación que demuestran los hidalgos y caballeros por las actividades de orden intelectual.

Para responder a éstas y otras preguntas, es necesario recurrir a una serie de fuentes bajo un doble criterio metodológico:

a. Por un lado, el análisis serio y riguroso de aquellas fuentes que directamente atañen al estudio positivista de la cuestión. Principalmente, nos referimos, entre otras, a los protocolos notariales en la búsqueda de testamentos, almonedas, alguna corresponencia e inventarios post mortem. Se trata, esta última, al decir de muchos, como una de las fuente más meritoria para 
rastrear la cultura escrita. Su estudio resulta sustancial para la confección de la deseada historia de la lectura en Extremadura. Urge examinar — como se ha hecho para Plasencia- los contenidos de las bibliotecas privadas por su valiosa e insustituible fuente de información; y no sólo de personajes nobles y hombres eminentes, sino también de caballeros, clérigos, letrados y artesanos sin importancia relevante. $Y$ esto a pesar de las dificultades para su elaboración dada la falta de precisión y la complejidad en la identificación de los títulos- y los defectos que una investigación sobre el fenómeno de la lectura basada únicamente en los inventarios tiene, dada la importante limitación metodológica: una cosa es tener libros y otra es leerlos. Además, el inventario resulta ser la tenencia de libros en un momento determinado: el de la muerte del poseedor, indicando seguramente los libros conservados y heredados de nuestros antepasados. Del mismo modo, un personaje puede haber leído cosas no presentes en su biblioteca, y, por consiguiente, no mencionadas en el inventario analizado. A pesar de todo, como decimos, su análisis es indispensable, y más para zonas como Extremadura caracterizadas por el dicho retraso historiográfico.

b. En segundo lugar, es ineludible el estudio de aquellas otras fuentes, que podríamos calificar como indirectas, de carácter histórico, literario y simbólico, y que son de utilidad para especificar la visión que de los libros y de la lectura se tenía en un determinado momento histórico. Son múltiples, y van desde las fuentes tradicionales o narrativas -que incluyen memorias, diarios, epistolarios, y correspondencia; autobiografías, papeles propagandísticos, gacetas, avisos y noticias; o relatos y memorias de viajeros)-, a obras de carácter jurídico —como los procesos judiciales-, literario (obras literarias, representaciones teatrales y piezas liminares); o simbólico-plástico - pensemos en la literatura emblemática- Dichas fuentes son especialmente convenientes para el analisisis de la visión -o percepción social- que de la cultura escrita se tenía en el Antiguo Régimen, dando multitud de interrogantes sobre su función, el uso cotidiano de los documentos o las prácticas de lectura.

En definitiva, el SIH que proponemos necesitaría para su realización del análisis pluridimensional de la historia del libro y de la lectura.

2.2. Los sistemas de información históricos (SIH): objetivos, necesidad y problemática

Dado que hemos establecido ya que el principal objetivo de este trabajo gira en torno a la documentación aplicada la investigación histórica del
Libro y la Lectura, no podemos dejar de estudiar el documento considerado como fuente de información. En este sentido, los sistemas de información histórica y documental estudian y analizan los documentos para extraer información histórica. Por todo ello, parece preciso hacer una breve aproximación al concepto de dichos sistemas de información.

\subsubsection{Los sistemas de información documental}

Un sistema de información documental está constituido por un conjunto de subsistemas que se relacionan entre sí para lograr un objetivo común, si uno de los susbsistemas falla, se altera el sistema total. El sistema de información documental puede ser visto en dos niveles: el primero puede ser la biblioteca en sí, donde los subsistemas están constituidos por los diferentes procesos que se realizan en ella. Por ejemplo, un sistema integrado documental estaría diseñado por: préstamo interbibliotecario, OPAC, catalogación y control de autoridad, circulación y préstamo, desarrollo de colecciones, publicaciones periódicas, todo ello alimentando la base de datos maestra que puede ser manual o computarizada.

Un sistema de información documental computarizado proporciona mayor capacidad para el procesamiento de datos en información que sirven para tomar decisiones. Cada uno de los subsistemas hace sus propios procesos y alimenta el archivo maestro del Sistema: la gran base de datos integrada. Un sistema de información documental Integrado permite además un mayor acceso a la información, minimiza la redundancia de datos, facilitando que todos los elementos, datos y registros se relacionen entre sí. El sistema de información documental asegura el asequible flujo de la información, para ello debe formular políticas y fomentar el proceso de comunicación con los usuarios del Sistema. Hoy por hoy, los sistemas de información documental han asumido el papel central del manejo de la información de sus organizaciones y el diseño de sistemas de acceso.

\subsubsection{Los sistemas de información histórica}

A juicio de García Marco (1995), los SIH nacen como "una solución integradora: como un interfaz entre el tratamiento meramente archivístico y el análisis del dato histórico que ayude a optimizar el flujo de la información en el campo de las ciencias sociales y humanas", y su objetivo es resolver, de forma documental, las necesidades de los usuarios de información histórica. Esto es, los SIH resultan ser un instrumento operativo que convierte los documentos históricos debidamente conservados en información, que previamente, tratada mediante los diferen- 
tes tratamientos documentales, es utilizada por el investigador para la producción de nueva información.

En este orden de cosas, el archivo histórico es un potencial centro de documentación y $\mathrm{SIH}$, sistema que a su vez labora por obtener de modo eficaz las notas de conservación, accesibilidad y difusión de la información histórica mediante operaciones de representación y organización de la información contenida en los documentos y que, finalmente, da a luz productos informativos propios como son las bases de datos históricas o facilita el acceso a bases de datos históricas producidas por otros sistemas de información.

En suma, los SIH se configuran como las herramientas más logradas en el campo que nos ocupa. Se hacen posibles merced al uso de las tecnologías informáticas e integran bases de datos y otros procedimientos similares que aseguren el correcto flujo de la información para la Historia. Desde las importantes reflexiones que García Marco (1995) ha dedicado a esta cuestión, procede enumerar las cuestiones de mayor interés relacionadas con los sistemas de información histórica. Estos sistemas:

a. Estructuran información en forma de bases de datos de carácter referencial, cuantitativo, textual, factual y multimedia.

b. Evitan la duplicación de tareas de recopilación y organización documental en tanto en cuanto la información estructurada es accesible, conectable e incorporable a otros SIH.

c. Propician la especialización de la documentación histórica por parte de las instituciones responsables de la construcción de tales sistemas.

d. Contribuyen a normalizar la ciencia histórica y su crecimiento en cuanto éste "es modelado como producto de la interacción de tres polos diferentes:

\section{d.1. la fuente histórica;}

d.2. la Historiografía, es decir, las interpretaciones históricas realizadas por los diferentes historiadores que han existido; $y$

d.3. el trabajo del investigador actual que es, entre otras cosas, consecuencia de su formación y filiación científica, de su punto de vista teórico y de su conocimiento de las fuentes, de los trabajos anteriores de otros investigadores y de la metodología".

e. Tratan de integrar todos los elementos referidos a las fuentes y a los resultados de las investigaciones, lo que incluye la normalización de las operaciones documentales y de la terminología, entre otros factores.

Un SIH no es una simple colección de fuentes o una descripción de unos determinados fondos, aunque lleven añadidas su digitalización y su etiquetado. Aunque dichas colecciones y fondos digitales constituyen componentes naturales de un $\mathrm{SIH}$, éste es, ante todo, la integración en un sistema informático de fuentes primarias y secundarias, datos y procedimientos de explotación bajo el paraguas de presupuestos teóricos y metodológicos compartidos por una comunidad de investigadores. De la misma manera, tampoco es un SIH la simple aplicación de herramientas informáticas a la investigación sobre el pasado. Un SIH es la aplicación sistemática de la informática a la investigación sobre el pasado, partiendo de instituciones y grupos de investigación comprometidos en compartir sus fuentes, datos y procedimientos, aunque, lógicamente, puedan disentir en el uso y conclusiones que saquen a partir del $\mathrm{SIH}$.

Diversas experiencias realizadas en el campo como la de García Marco (1995) nos muestran que el impulso más importante no cabe esperarlo de la demanda -esto es, de los investigadores- sino de las instituciones que les ofertan información y que están siendo animadas, desde las organizaciones que las encuadran y por el propio compromiso de sus miembros, por políticas cada vez más atentas al conocimiento, conservación y puesta en valor del patrimonio cultural y natural. Esta preocupación por el patrimonio entronca con la atención creciente que las elites y aún las masas de las sociedades posmodernas prestan al pasado. El pasado ya no es tan sólo una fuente de conocimiento para la comprensión de las leyes de la naturaleza y de las sociedades y, por tanto, para el desempeño humano eficaz - lo cual, aunque evidente, resulta demasiado abstracto para la gran parte de la población-, sino sobre todo una fuente de entretenimiento, cultura e identidad, aspectos fundamentales en cualquier sociedad sana.

Los SIH se sitúan en el centro de algunos de los principales énfasis -y tensiones, en la medida en que constituyen fuerzas muchas veces enfrentadas- de esta década: la tecnología, la identidad, la integración, la diversidad, la acumulación cultural, la globalización y el cambio cultural. Así, por un lado, los registros de hechos "pasados» constituyen la fuente de conocimiento por excelencia en todas las disciplinas. Por el otro, la acumulación de información -en la actualidad extraordinariamente facilitada y abaratada gracias a las nuevas tecnologías de la información- permite su análisis posterior desde nuevos puntos de vista, con 
otros objetivos y mediante nuevos enfoques metodológicos. Finalmente, el creciente interés actual en el patrimonio cultural —facilitado por el desarrollo económico y exigido por la necesidad de mantener anclajes en un entorno social sometido a procesos acelerados de cambionecesita de instrumentos avanzados de gestión. Ciertamente, los sistemas de información histórica se sitúan en el centro de todos estos procesos al servicio de una gestión eficaz del patrimonio cultural en todas sus vertientes.

Podemos concluir, por tanto, que la construcción de SIH no es un problema tecnológico, puesto que disponemos de todas las herramientas necesarias para ello: Internet, gestores de bases de datos universales, SGML y sus variantes, etc. El problema de los SIH es un problema político, a saber, el problema de organizar a los responsables de las políticas, de los repositorios y de las investigaciones histórico-culturales para crear entornos de gestión integrada del conocimiento sobre el pasado.

Dado que las ciencias históricas y las industrias culturales se van a beneficiar extraordinariamente de cualquier iniciativa en este sentido, aliar los intereses de la investigación histórica, por un lado, y de la gestión de los vestigios del pasado, por el otro, constituye la estrategia de elección para la potenciación y desarrollo de los $\mathrm{SIH}$, un concepto que espera que, por fin, llegue su momento.

\section{Justificación}

El interés por el pasado no es mera curiosidad, sino un signo de nuestros tiempos. Las sociedades posmodernas vienen prestando interés creciente al pasado, puesto que su consideración es básica para dos de sus énfasis: el conocimiento y la gestión del cambio permanente. El interés que despierta actualmente el conocimiento de nuestras raíces culturales ha provocado que en estos últimos años se contemple con respeto y atención todo lo relativo a ellas. Parece que últimamente se ha dado un "nuevo nacionalismo", en el sentido más cultural, que consiste en una vuelta de nuestra atención hacia nuestros orígenes, nuestra cultura, "Io nuestro", tanto a nivel nacional como regional, y esto se traduce en una multitud de planes, proyectos y medidas que fomenten el desarrollo de nuestra identidad. El entorno internacional y nacional es favorable, pues la globalización ha acarreado este interés por las culturas locales. Por ello, es lícito explorar nuestras raíces para así construir nuestro futuro.

La Cultura Escrita perteneciente a nuestra región forma parte esencial de esas raíces cultu- rales que tenemos que descubrir y conservar, ya que, como parte integrante del Patrimonio Cultural, es un poderoso medio de acercamiento de los pueblos y de afirmación de su identidad cultural, ya sea a través de sus manifestaciones "tangibles" o "intangibles". La Cultura Escrita tiene una enorme importancia social, cultural y política en el papel de la historia de los pueblos, y por ello ocupa un lugar significativo en la cultura contemporánea. Los valores y tesoros que la componen nos dan pistas sobre nuestro pasado y no deben perderse en el presente. Ciertamente, la Cultura Escrita es una forma de patrimonio sui generis a caballo entre el patrimonio material e inmaterial, pues debe tratar con aspectos tangibles, tales como el mundo de la edición o de la imprenta, y del mismo modo atender a aspectos mucho más intangibles, como la cultura literaria.

Dentro de la Cultura Escrita, la historia del libro y de la Lectura en todos sus aspectos (materiales, sociales y culturales), toma una relevancia fundamental, tanta que en la historia del estudio de esta disciplina (Cultura Escrita), evoluciona desde sus inicios de ser una materia únicamente relacionada con el alfabetismo y la paleografía como disciplina auxiliar a ser una historia del libro y la lectura en el sentido más amplio de las relaciones históricas entre la sociedad y la escritura o la lectura.

De hecho, la historia de la lectura y la escritura siempre ha revelado la vinculación de estas prácticas al poder social y político, como ya ocurriera desde los escribas egipcios. En todo caso, el estudio de estos aspectos roza más la consideración de lo que se llama "patrimonio intangible" y hay que considerar en ella aspectos históricos, sociológicos y de toda índole. Es por eso que debemos prestar una especial atención a este tipo de información, para poder conocer, entre otras cosas los diferentes usos de la lectura y la escritura y sus alcances sociales, culturales, políticos, económicos e incluso antropológicos. Este tipo de conocimientos se traduciría en una mejor comprensión de nuestra cultura actual, ya que la lectura es parte indispensable de la cultura de cualquier medio.

De todo ello se desprende la necesidad de localizar y conservar toda la información relativa a la historia del libro y de la lectura de nuestra región, para que podamos entender qué leían nuestros antepasados y qué leemos ahora, cómo han evolucionado nuestros hábitos y principios de lectura, cómo ha avanzado la industria del libro y su interés como mercado, y, especialmente, inferir la trascendencia que tienen todos esos aspectos sobre nuestra cultura y 
nuestra identidad como pueblo y como región. Entre otras cosas, somos lo que leemos.

Pero, para que podamos estudiar la información que hallemos relativa a esta materia, no basta sólo localizarla y conservarla. Una mera conservación de información de la Historia de la Lectura mal organizada no reportaría los beneficios en materia de investigación que se pretende obtener con ello, y en muchos casos supondría precisamente el extravío (es decir, la pérdida) de valores patrimoniales que se quieran conservar, de igual modo que un libro mal catalogado supone su extravío en una biblioteca. Por tanto, una correcta conservación implica su correcta organización.

Se conoce que, aunque hay mucha documentación teórica para el estudio de la historia del libro y la lectura durante el último siglo, no ocurre lo mismo con las fuentes de información para su estudio. Normalmente los investigadores suelen circunscribirse a las fuentes de información directas (que suelen ser escasas e incompletas), olvidándose de otras muchas fuentes porque no son tan sistemáticas, aunque en muchas ocasiones contengan datos relevantes que es necesario explicitar mediante instrumentos de extracción de contenido. Es por esto que tales fuentes de información indirectas, como por ejemplo las obras literarias, quedan muchas veces relegadas al olvido.

Sistematizando, consideramos la necesidad de un SIH sobre la historia del libro y la lectura en Extremadura por varios motivos:

1. La importancia histórico-social de dicha disciplina, y la escasa sistematización que ha tenido hasta ahora como materia de investigación.

2. La necesidad de una correcta extracción, análisis y conservación de la información relativa a este campo para facilitar el acceso a su estudio y para evitar su desaparición.

3. La urgencia de organizar todas las manifestaciones de esta disciplina, tanto explícitas en estudios e investigaciones directas; como implícitas en otro tipo de fuentes de información.

4. El desarrollo de herramientas documentales que nos permitan analizar correctamente toda la información existente sobre la historia del libro y la lectura en nuestra región.

5. Y, por último, el interés de difundir las peculiaridades de la idiosincrasia lectora de los extremeños en el pasado y en el presente, con objeto de obtener una perspectiva más profunda de nuestras raíces culturales.

\section{Pasos para la elaboración}

A continuación, se facilitará una serie de pautas que constituirían la base teórica y conceptual para la creación del Sistema de Información citado sobre la historia del libro y la lectura en Extremadura. Tales etapas o pasos son señalados en sentido meramente orientativo, siendo así que se pueden introducir más pasos o simplificar otros, dependiendo de la documentación que tengamos como base en el trabajo de campo, la materia que queramos tratar, etc.

Asimismo, y aunque se ha mencionado escuetamente en la introducción, haremos alusión a las metodologías que emplearemos para ello:

a. Técnicas documentales: dichas técnicas nos permitirán el estudio de diferentes documentos y obras como fuente de información. Estas técnicas se fundamentan sobre todo en el análisis de contenido de dichas obras, pudiéndose hacer tanto de forma cuantitativa como cualitativa, empleándose las diferentes técnicas de análisis documental existentes. Destacar que, según los casos, también se necesitará prestar atención a la información implícita en las fuentes y no sólo explícita, para lo cual las técnicas de análisis cuantitativo aún no están del todo perfeccionadas, pese a los avances en el campo. La aplicación de técnicas documentales dará lugar a la creación de herramientas documentales como un catálogo documental, unos índices toponomásticos y un tesauro ad hoc como lenguaje documental específico para la temática.

b. Análisis crítico de la documentación empleada, que nos daría una visión global de la evolución histórico-social de las materias objeto de tal corpus documental, y que mostraría las características generales e históricas de la historia del libro y la lectura y su evolución.

c. Y el empleo de las nuevas tecnologías de la información, que darán lugar a la confección un gestor documental automatizado aplicable a la temática en cuestión, y que permitirá la consulta y recuperación de la formación de forma pertinente y eficaz.

Utilizando estas técnicas, podremos llevar a cabo las siguientes tareas:

1. Recopilación de bibliografía general y especializada sobre historia del libro y la lectura, y su estudio (tanto de manera aislada como en conjunto) para sacar ideas y datos sobre un estado de la cuestión que podamos extrapolar a la investigación sobre el tema en Extremadura. Dicha bibliografía podrá tener la extensión y el alcance que queramos, teniendo en cuenta los criterios de exhaustividad, precisión y adecua- 
ción en cuanto al tiempo y recursos de que dispongamos y la amplitud que queramos dar a nuestro Sistema de Información.

2. Trabajo de campo, orientado hacia la extracción manual e intuitiva de:

2.1. cualquier referencia específica relativa a algún aspecto sobre la historia del libro y la lectura en Extremadura, siendo el conjunto de referencias destinado a formar un catálogo de ellas que formarían parte de una futura base de datos con interrelaciones con las demás herramientas documentales. Para ello, los fragmentos deben ir recopilando en una base de datos simple, en Access, Filemaker o cualquier otra aplicación al efecto, y deben ir indizando con términos tanto de materias como toponomásticos, así como llamadas o reemisiones a la localización física de la información.

2.2. Ios términos que condensan el contenido sobre la historia del libro y la lectura en Extremadura dentro de las diferentes obras y más concretamente dentro de las mencionadas referencias (los utilizados para indización por materias), términos que acabarán convirtiéndose en la base para crear los descriptores de un tesauro que se utilizaría como herramienta metodológica.

3. Contrastar críticamente los datos obtenidos del estudio de la bibliografía con los derivados del trabajo de campo, obteniendo un análisis de las referencias en torno a las principales categorías establecidas (categorías que corresponden a los macrodescriptores del tesauro).

4. Elaborar fuentes documentales derivadas de estos datos, tales como:

4.1. Catálogo con referencias de las fuentes documentales relativas a todos los aspectos de la historia del libro y la lectura en Extremadura (selección de textos), con vistas a establecer una futura base de datos. Después de la elaboración del tesauro, se volvería a este catálogo para su correcta indización con descriptores.

4.2. Índices onomásticos y topográficos de todos los nombres propios y autoridades que aparecen en dichas referencias.

4.3. Microtesauro de descriptores ad hoc: es decir, específicamente creado para poder indizar estas referencias relativas a la historia del libro y la lectura en Extremadura dentro de la documentación empleada.

5. Integrar estas tres fuentes documentales dentro de un Sistema de Información integrado con base automatizada, que permitiría una recuperación exhaustiva de referencias dentro de la documentación empleada.
En este caso, obtendríamos una base de datos automatizada y sistemática con las referencias a la historia del libro y la lectura, las cuales estarían indizadas, por un lado, con descriptores de materia que se obtendrían del tesauro y, por otro, con las autoridades del fichero conformado para dicho propósito. Ambas herramientas documentales (tesauro y fichero de autoridades) estarían interrelacionadas de manera automatizada dentro del soporte con la base de datos de referencias, lo cual repercutiría en una mayor facilidad por parte del usuario en la recuperación de las referencias tras la búsqueda de un descriptor o autoridad concretos que éste reclamara en su búsqueda dentro del tesauro o fichero, incluso de manera simultánea o combinada. La idea sería que en dicho Sistema de Información el usuario podría desplegar menús con los descriptores del tesauro y/o autoridades del fichero y combinarlos para hallar mediante búsquedas booleanas las referencias de la base de datos que correspondan a sus necesidades, habiendo sido previamente esas referencias indizadas según los descriptores del tesauro y autoridades del fichero.

\section{Herramientas obtenidas del proceso}

El resultado de la aplicación de las técnicas documentales al trabajo de campo efectuado sobre la diferente documentación que conforma nuestras fuentes de información se materializa en la creación de una serie de herramientas, lo cual es esencial para demostrar que la aplicación de técnicas documentales a estas fuentes puede devolver un alto valor informativo.

Cabe destacar que las dos primeras (catálogo de referencias e índices onomásticos) se han extraído de forma más intuitiva y conceptual, mientras que la tercera (tesauro o mejor dicho microtesauro de descriptores) es la herramienta documental que normalizará las dos primeras, permitiendo su integración en una macroherramienta documental como podría ser un sistema de información automatizado, el cual conformaría las tres herramientas y las que quisiéramos añadir. En otros términos, se constata la necesidad de una herramienta de lenguaje documental como el tesauro para interrelacionar todo el sistema. En este sentido, el tesauro tiene por finalidad la doble pretensión de una homogeneización y normalización terminológica en razón de la temática o disciplina que aborde, y en segundo lugar de favorecer la sistematización del proceso de documentación con la finalidad de una fácil recuperación de la información acumulada. Cuando se aplica a la cultura, y en particular a la historia del libro y la lectura, no debemos olvidar que la propia consideración de 
que lo que forma parte de esta historia del libro y la lectura no sólo ha sido y es cambiante en cuanto a sus contenidos materializables sino en los valores sociales a partir de los cuales se define esta cultura.

Por lo dicho, veamos de forma sistematizada dichas herramientas documentales, que son las siguientes:

\subsection{Catálogo de referencias}

La primera tarea en la que nos enfrentamos en nuestro trabajo de campo para determinar el valor contenido de la historia del libro y la lectura es determinar qué partes de la obra contienen alguna referencia a esa materia. Para ello, procederemos a la lectura comprensiva y analítica de la obra para poder realizar una extracción sistemática de dichas partes. A esas partes se las denominó referencias porque hacían referencia o alusión a uno o más aspectos de la historia del libro y la lectura en Extremadura. No se debe confundir esta acepción del término con el de referencia o cita bibliográfica en el sentido de remisión a la obra de otro autor. Asimismo, catálogo tiene aquí más sentido de repertorio que la acepción, conocida por los bibliotecarios y documentalistas, de un registro con las fichas bibliográficas de los títulos de una biblioteca.

La tarea de la extracción de dichas referencias resulta un trabajo de campo, si bien interesante, también largo y con cierta complejidad, ya que se trata de una labor muy conceptual y principalmente intuitiva del documentalista, y por tanto presenta problemas de subjetividad. Se debe ser muy estricto para distinguir lo que se busca dentro de la obra y prestar atención a todas las formas en que la historia del libro y la lectura se manifestará dentro de la documentación empleada. La extracción de las referencias se abordará tras la lectura conceptualizada y comprensiva de la obra. Se irían registrando en una base de datos realizada en Access, Filemaker o la aplicación de creación de bases de datos preferida. Los registros de dicha base de datos contendrían los siguientes campos:

a. N_ref: Número de registro de la referencia (número currens).

b. Resumen: Pequeño resumen o sinopsis de la acción del fragmento. En ocasiones, se acompaña de una breve explicación o análisis textual que encontramos dentro de la edición que estamos examinando.

c. Texto: El fragmento de la obra en sí, con unos criterios lógicos de extensión. d. Localización: Una llamada o reemisión al documento físico donde se encuentra el fragmento registrado.

e. Descriptores de contenido, dispuestos de forma alfabética cuando hubiera más de uno dentro de la misma categoría. Dentro de éstos, tenemos:

e.1. Descriptores onomásticos (de nombres, buscando la máxima normalización posible). De éstos, hemos determinado incluir tres tipos:

e.1.1. Desc_Onom: De personas, aunque si así se decide pueden consignarse también títulos de obras literarias $u$ otros nombres que no procedan en otros campos.

\section{e.1.2. Desc_Topog: De lugares.}

\section{e.1.3. Desc_Ent: De entidades.}

e.2. Descriptores de materia (Desc_Materia). En un primer momento, éstos serían términos en lenguaje natural hallados de forma intuitiva tras condensar conceptualmente el contenido del fragmento. Sin embargo, dado que este trabajo intuitivo y manual y el uso del lenguaje natural traen problemas como la ambigüedad, la sinonimia, etc.; en una fase posterior del trabajo, y ya tras haber creado el tesauro de descriptores -el cual detallaremos más adelante-, estos términos serían sustituidos por los descriptores del tesauro que coincidan conceptualmente con esos términos en lenguaje natural, con lo que la indización resulta así mucho más pertinente y exacta.

En este caso, la base de datos aún no tiene, por ahora, interrelación con el resto de herramientas documentales (índice toponomástico y tesauro). Esto, como se explicará, se podrá hacer en el futuro mediante el cargado del tesauro y de los índices toponomásticos dentro de bases de datos similares o en otras aplicaciones compatibles (si esto no fuera posible, se contempla la exportación de esta base de datos a otra aplicación donde se puedan cargar las herramientas mencionadas sin problemas). Sin embargo, las diferentes aplicaciones de bases de datos hacen búsquedas simples de términos dentro de los campos que se quiera, lo cual implica poder buscar descriptores posicionándonos en el campo Desc_Materia.

Por cierto que en una futura base de datos automatizada que incluyera el texto completo de la obra, no haría falta, como se ha hecho hasta ahora, "cortar" simplemente los fragmentos para incluir cada uno en un registro. Usando las interrelaciones con las que cuentan incluso aplicaciones de bases de datos simples como Access o Filemaker podría llegarse incluso a hacer se- 
lecciones dentro del texto completo, eliminando así el problema de tener que limitar el número o la extensión de los fragmentos dentro de cada registro por problemas de espacio. De todas formas, ésta es una tarea ingente y compleja por implicar cierto manejo desenvuelto de tales aplicaciones informáticas que queda para futuras investigaciones.

\section{2. Índices toponomásticos}

Los índices toponomásticos (a menudo figurados en otras obras en los Apéndices) tienen una gran utilidad para que el investigador pueda "moverse" rápidamente por la obra, ya que, al igual que la recuperación mediante descriptores de un tesauro facilita que se puedan hallar fácilmente todas las referencias a un mismo concepto de contenido, un índice onomástico también remite con rapidez a todas las referencias que en la obra se encuentren respecto a una persona, entidad, lugar, etc. No son tan conocidas como herramienta documental propiamente dicha (ya que no forman parte de ni se construyen a través de lenguajes documentales), pero en este trabajo se determinará darle una importancia similar a herramientas documentales aceptadas por todos como el tesauro ya que pasan un proceso similar al documental (extracción intuitiva y posterior normalización de forma) $y$, ante todo, porque resultan de muy parecido valor informativo para el usuario ya que condensa contenidos y remite a la parte del texto que le es útil, ahorrándole un tremendo trabajo conceptual.

Los índices se harían partiendo de los campos de descriptores onomásticos de la base de datos del catálogo de referencias (excepto los de materias, los cuales servirán de base para el tesauro). Después de eso, los nombres tanto de personas y entidades, como de títulos de obras y lugares, serán normalizados de acuerdo con las normas vigentes. Todo ello resultarían 3 tipos de índices: índice onomástico de personas y entidades; índice topográfico, u onomástico de lugares; e índice de títulos de obras literarias. En estos índices se añadirían las llamadas o reemisiones a la situación física dentro del documento del fragmento donde aparece el nombre. Asimismo, y para completar la información derivada del índice, pueden contener algunos breves datos en relación a la entrada, e incluso el reenvío a otras asociadas o relacionadas con ella.

\subsection{Microtesauro de descriptores ad hoc}

Sin embargo, la herramienta documental que verdaderamente aglutinará el esfuerzo realizado en las dos anteriores es el tesauro, ya que el éste es un lenguaje documental y su empleo, como venimos diciendo, normalizaría todo intento de análisis de contenido de la obra. Éste es el resultado más importante del esfuerzo documental realizado.

Debemos puntualizar de manera significativa que no es nuestro objetivo elaborar un tesauro conceptual de toda la historia del libro y la lectura global, sino un Microtesauro específico (ad hoc) de la historia del libro y la lectura en Extremadura, y delimitado por la documentación en la que nos hayamos basado, por lo que la profundidad de éste dependerá de la amplitud y precisión de la documentación que hayamos escogido y los conceptos y términos descriptores de materias que hayamos extraído de ella.

Una vez determinados los criterios de alcance de nuestro tesauro y los componentes conceptuales en los que lo vamos a basar, procederemos a su elaboración. No entraremos en detalles sobre la metodología de construcción de tesauros porque es un tema en el que se ha profundizado ampliamente en muchos manuales y artículos, pero podemos resumir los siguientes pasos a hacer:

a. Delimitación de la profundidad y extensión del campo terminológico, trabajando sobre un área cerrada para que su construcción sea posible. En este caso, la historia del libro y la lectura en Extremadura.

b. Selección de la terminología, haciendo una primera selección intuitiva de los términos que condensaban el contenido de las referencias relativas a historia del libro y la lectura dentro de la documentación seleccionada. Esta documentación estaría compuesta tanto de fuentes directas (tales como inventarios, listas de libros, etc.) como indirectas (obras literarias, relatos y memorias de viajeros, etc.) que hicieran referencia al tema.

c. Determinación de campos temáticos y distribución de descriptores, creando una estructura jerárquica del tesauro, que presenta sistemática y sintéticamente toda la información que hay en el mismo. Sobre la selección de términos que mencionamos en el punto $b$, trataremos de obtener los descriptores, seleccionando el término más correcto para representar el concepto, normalizando su forma y estableciendo las primeras relaciones.

d. Establecimiento de las relaciones de equivalencia, determinando los términos preferenciales a través de la localización de sinónimos dentro y entre los campos. En esta fase disminuye de nuevo el número de términos y se plantean todas las posibles relaciones semánticas de cada uno de los descriptores y conceptos que van a 
conformar la estructura del tesauro, eliminando repeticiones.

e. Redacción de notas aclaratorias, definiendo con precisión el significado u otros datos necesarios de los términos. En caso necesario, se utilizarán instrumentos de referencia como el Diccionario de la Real Academia o la enciclopedia Encarta.

f. Establecimiento de relaciones jerárquicas, con un único árbol de relaciones jerárquicas entre todos los descriptores.

g. Establecimiento de relaciones asociativas, dado que existen relaciones de afinidad semántica que no suponen sinonimia ni jerarquía, se trata de una relación de afinidad semántica. Esta relación y la asociativa son las más conceptuales e intuitivas de todas y como tales implican una gran subjetividad, agravando la dificultad de establecerlas con corrección.

Una vez determinado el esquema con la selección de términos y sus correspondientes relaciones, se realizará el cargado de todos ellos en un programa gestor de tesauros, del cual se exportarán después las distintas presentaciones. El tesauro, como lenguaje vivo, debe ser adaptado a los cambios y reajustes de vocabulario y estructura. El crecimiento de los registros de la base de datos y la retroalimentación de los usuarios son el incentivo para su modificación. Por tanto, se trata de un instrumento que debe ser modificado y perfeccionado con la finalidad de abarcar cada vez más términos y una mayor facilidad de utilización por parte del usuario.

\section{Conclusiones}

Después de realizada la investigación, efectuados el trabajo de campo, el análisis conceptual del contenido de las diferentes fuentes de información y la aplicación a éste de las técnicas documentales, y desarrolladas como consecuencia de ésta la serie de herramientas documentales descritas, hemos sentado las bases documentales para la creación de un sistema de información documental para la investigación de aspectos relativos a la historia del libro y la lectura en Extremadura. En un futuro, estas bases confluirán en un futuro en un sistema de información integrado sobre el tema, cristalizando en unas herramientas que, algo más ampliadas y evolucionadas, formarán parte de un gestor documental que será de una enorme ayuda para todo aquel que pretenda investigar cualquier aspecto de historia del libro y la lectura en Extremadura.

Por tanto, destacamos entre las conclusiones obtenidas de dicho estudio las siguientes:
1. Se infiere de la aplicación práctica de la metodología presentada que la aplicación de las técnicas documentales para el análisis de la historia del libro y la lectura tiene una gran utilidad, ya que concluyen en la creación de herramientas de análisis de contenido que nos permiten condensar el contenido relativo a este campo en todo tipo de documentos, incluso en las fuentes indirectas. Con el uso de un Sistema de Información como el que se plantearía aumentarían las posibilidades de poder investigar sobre la materia con información sintetizada y sistematizada de antemano, con lo que queda demostrado que la aplicación de herramientas documentales sobre fuentes indirectas como obras literarias (esto sirve para todo tipo de fuentes de información, pero vemos que en las indirectas ocurre con mayor intensidad) multiplica el valor informativo de la fuente como recurso para acceder a información de primera mano.

2. Se destacan las posibilidades de adaptación o implantación de un Sistema de Información sobre la historia del libro y la lectura en Extremadura como herramienta documental para estudiar este campo. Asimismo, independientemente del aspecto de la historia del libro y la lectura que se estudie, es interesante que los investigadores conozcan las ventajas reales que tiene la implantación de un tesauro idóneo y ya desarrollado dentro de éste, tanto considerando cada sistema de información aisladamente como el conjunto de los sistemas españoles que podrían relacionarse a través de este instrumento de control terminológico.

$\mathrm{Si}$, como se ha señalado en repetidas ocasiones, en último extremo la función del tesauro es facilitar la comunicación o diálogo entre usuarios y sistemas, también su carácter mediador o instrumental es fundamental para relacionar varios sistemas entre sí. La interconexión de sistemas puede utilizarse para facilitar la labor de servicios de información especializados. Por este motivo, la realización de esta investigación ofrece diversas oportunidades futuras:

1. Aplicar un futuro tesauro común facilitaría la creación de redes de información sobre la historia del libro y la lectura y la búsqueda e intercambio de información en un contexto global de redes de información al que no podemos sustraernos en la época de Internet. La capacidad potencial del tesauro que se presenta como base para la un sistema de información documental integrado sobre la historia del libro y la lectura o para ser implantado totalmente en otros sistemas de información, abre una perspectiva para el estudio de la historia del libro y la lectura a través de herramientas documentales. 
2. Por otro lado, una investigación, por rigurosa que sea, nunca deja cerradas todas sus opciones de mejora, siempre es susceptible de ser completada y perfeccionada. Por tanto, este estudio abriría nuevas líneas de trabajo, la más importante de las cuales es la opción de integrar las tres herramientas conceptuales concebidas a partir de este trabajo (tras su ampliación y contextualización) dentro de un sistema de información documental integrado y automatizado sobre la historia del libro y la lectura en Extremadura, el cual consistiría en un gestor documental automatizado, mediante el cual conseguiríamos, por un lado, el acceso a la información relativa a cada documento del catálogo de manera sencilla y rápida, y por otro, y a través del tesauro, estableceremos un sistema automatizado de búsqueda y recuperación de la información.

En resumen, en este trabajo se ha demostrado que es posible la consideración sistemática de todos estos elementos relativos a historia del libro y la lectura existentes en las diferentes fuentes de información explícita e implícitamente (y por tanto, difíciles de constatar a priori) y su registro, en la medida de lo posible, a través de técnicas documentales ad hoc, con el objetivo básico de habilitar una serie de instrumentos que ayuden a crear un sistema de información para el estudio de la historia del libro y la lectura en Extremadura.

\section{Notas}

(1) Es fundamental para nuestro trabajo la obra de R. Chartier. Vid. entre otros muchos títulos para nuestro cometido aquí Practiques de la Lecture, Rivages, Marsella, 1985; Lectures et lecteurs dans la France d'Ancien Regime, Seuil, París, 1987; Les usages de l'imprimé, Fayard, París, 1987; Cultural History Between Practiques and Representations, Cambridge, 1988; o "Livres bleus et lectures populaires. Mi XVII siècle-debut XIX" en Culturas populares: diferencias, divergencias, conflictos, en Europa entre los siglos XVI y XVII, Gedisa, Barcelona, 1995. Al decir de Ricardo García Cárcel, Roger Chartier es, sin duda, el historiador que más ha influido en la proyección de la cultura en los últimos diez años. Tras un inicial interés en los años setenta por la problemática de la historia de la educación, desenvolviéndose en sus primeros escarceos metodológicos bajo la sombra de la Escuela de Annales irrumpe en el horizonte historiográfico de modo bien visible en los años ochenta, a través de la dirección del III volumen de la Historia de la Vida Privada -1985-y sobre todo de la codirección, junto a H.J. Martín de la Histoire de l'edition française -3 vols., 1982, 1984 y 1985, respectivamente. Para este apartado seguimos a Le Goff, J.; Chartier, R.; y Revel, J. Diccionario del saber moderno. La Nueva Historia. Bilbao: Ed. Mensajero, 1988 (voz Libro, pp. 91 y ss).
(2) Vid. Testón Núñez, Isabel: "Lectura y Mentalidad en Cáceres en el siglo XVII" Norba: Revista de Arte, Geografía e Historia, Universidad de Extremadura, Cáceres, 1980. Pág. 299-337; Santillana Pérez, Mercedes. La vida: nacimiento, matrimonio y muerte en el partido de Cáceres en el siglo XVIII. Cáceres: Institución Cultural el Broncese, 1992; Aragón Mateos, Santiago. La nobleza extremeña en el siglo XVIII. Mérida: Consejo Ciudadano de la Biblioteca Pública Municipal Juan Pablo Forner, 1990; Marcos Álvarez, F. y Cortés Cortés, F.: Educación y analfabetismo en la Extremadura meridional (s. XVII), Servicio de Publicaciones de la Universidad de Extremadura, Cáceres, 1987, Vivas Moreno, A. "De la historia del libro a la historia de la lectura. Introducción a un informe sobre la historia cultural en la Extremadura del Antiguo Régimen" en El Humanismo Extremeño. I Jornadas. Zafra. Fregenal de la Sierra: Rea Academia de Extremadura de las Letras y las Artes, 1996, pp. 387-400.

(3)Le Flem, J.P.: "Cáceres, Plasencia y trujillo en la segunda mitad del siglo XVI (1557-1596)". Cuadernos de Historia de España, 1967, pp. 248-299; Fernández Nieva, J. "Censura e índice de libros prohibidos en Extremadura" Revista de Estudios Extremeños, 39, 2, 1983, pp. 221-284; Santillana Pérez, Mercedes. "Estado de la instrucción pública en la diócesis de Coria a comienzos del s. XIX" Norba. Revista de Historia, 11-12, 1991-1992, pp. 251-262; Rodríguez Cancho, M. La información y el estado: La necesidad de interrogar a los gobernados a finales del Antiguo Régimen. Cáceres: Universidad de Extremadura, 1992, y de mismo autor "Interrogatorios del s. XVIII. Estudio comparativo" Norba, II, 1981, pp. 56-71.

\section{Referencias}

Chevalier, Maxime (1976). La Celestina según sus lectores en Lectura y lectores en la España del siglo XVI y XVII. Madrid: Ed. Turnar, 1976. 138-167.

García Gómez, F.J. (2001). Información digital e investigación histórica: una aproximación. // Biblos. 9. http://www.bibliosperu.com/articulos/9/B9-02.pdf (200705-31)

García Marco, F.J. (1995) Los sistemas de información histórica: entre la Archivística y la Historia. // Ruiz Rodríguez, A. (Ed.). Manual de Archivística. Madrid: Síntesis. 103-184.

López Yepes, J. (1997). Los caminos de la información. Cómo buscar, seleccionar y organizar las fuentes de nuestra documentación personal. Madrid: Fragua.

López Yepes, J. (1998). Las bases de datos históricas en Anales de Documentación. Murcia: Universidad de Murcia. 1:99-125.

Luengo Pacheco, R. (2002). Libros y lectores en Plasencia (siglos XVI-XVIII). Cáceres: Universidad de Extremadura, Servicio de Publicaciones.

Vivas Moreno, A (2000). Sistemas de información histórica para las colecciones facticias de archivos históricos: modelo de investigación. // Multidoc. 10. http://www. ucm.es/info/multidoc/multidoc/revista/num10/paginas/pdf s/Avivas2.pdf (2007-05-31) 